\title{
Mecânica Clássica - Homogeneidade do Espaço
}

\author{
Mario Cezar Bertin ${ }^{1}$ \\ ${ }^{1}$ Instituto de Física, Universidade Federal da Bahia
}

September 24, 2020

Rich media available at https://youtu.be/Xv63t3aqu18

\section{Translações}

O espaço euclidiano tridimensional $\mathbb{R}^{3}$ é homogêneo, o que significa que ele tem as mesmas propriedades geométricas independentemente da posição da origem do sistema de coordenadas. A homogeneidade também significa a invariância da métrica por translações.

Podemos analisar a operação de translação de duas formas distintas. A forma ativa consiste em mudar a posição do sistema físico com relação ao observador, ou seja, a distância entre o observador e o sistema, sem mudar a orientação do sistema com relação aos eixos ordenados. A forma passiva consiste em mudar a posição da origem do sistema de coordenadas, sem modificar a orientação dos eixos ordenados em si. Se $x \in \mathbb{R}^{3}$ é a posição de uma partícula, uma translação ativa consiste em mudar a posição da partícula para um determinado ponto $y \in \mathbb{R}^{3}$, pela operação

$$
x \longrightarrow y:\left\{\begin{array}{l}
y_{1}=x_{1}+a_{1}, \\
y_{2}=x_{2}+a_{2}, \\
y_{3}=x_{3}+a_{3},
\end{array}\right.
$$

em que $a_{1}, a_{2}$ e $a_{3}$ são números reais denominados parâmetros da transformação. Por outro lado, uma translação passiva não modifica o sistema físico em si, mas apenas o sistema de coordenadas, o que equivale a mudar o observador de posição. Neste caso, a mesma transformação (1) tem a forma

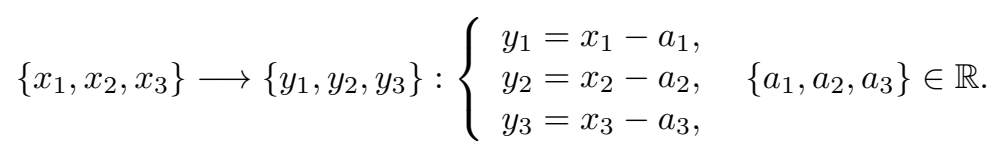

Note que a transformação ativa é a inversa da transformação passiva, para que ambas representem a mesma operação. Por enquanto, vamos abordar apenas a forma passiva das transformações de translação e rotação.

Note que (2) é uma transformação linear. Podemos coloca-la em uma forma matricial:

$$
\left(\begin{array}{c}
y_{1} \\
y_{2} \\
y_{3} \\
1
\end{array}\right)=\left(\begin{array}{cccc}
1 & 0 & 0 & -a_{1} \\
0 & 1 & 0 & -a_{2} \\
0 & 0 & 1 & -a_{3} \\
0 & 0 & 0 & 1
\end{array}\right)\left(\begin{array}{c}
x_{1} \\
x_{2} \\
x_{3} \\
1
\end{array}\right)=\left(\begin{array}{c}
x_{1}-a_{1} \\
x_{2}-a_{2} \\
x_{3}-a_{3} \\
1
\end{array}\right)
$$


em que

$$
T(a) \equiv\left(\begin{array}{cccc}
1 & 0 & 0 & -a_{1} \\
0 & 1 & 0 & -a_{2} \\
0 & 0 & 1 & -a_{3} \\
0 & 0 & 0 & 1
\end{array}\right)
$$

é a matriz de translação.

A forma matricial é útil para determinar algumas importantes propriedades das translações. Primeiro, duas translações em sequência formam uma única translação. Uma translação passiva com parâmetros $\left\{a_{1}, a_{2}, a_{3}\right\}$ seguida por outra translação de parâmetros $\left\{b_{1}, b_{2}, b_{3}\right\}$ representa uma única translação cujos parâmetros são dados por $\left\{a_{1}+b_{1}, a_{2}+b_{2}, a_{3}+b_{3}\right\}$. Para ver esta propriedade, vamos supor que o sistema de coordenadas $\{x\}$ seja transladado no sistema $\{y\}$ através da transformação (3). Depois, passamos ao sistema de coordenadas $\{z\}$ através da transformação

$$
\left(\begin{array}{c}
z_{1} \\
z_{2} \\
z_{3} \\
1
\end{array}\right)=\left(\begin{array}{cccc}
1 & 0 & 0 & -b_{1} \\
0 & 1 & 0 & -b_{2} \\
0 & 0 & 1 & -b_{3} \\
0 & 0 & 0 & 1
\end{array}\right)\left(\begin{array}{c}
y_{1} \\
y_{2} \\
y_{3} \\
1
\end{array}\right)
$$

em que

$$
T(b)=\left(\begin{array}{cccc}
1 & 0 & 0 & -b_{1} \\
0 & 1 & 0 & -b_{2} \\
0 & 0 & 1 & -b_{3} \\
0 & 0 & 0 & 1
\end{array}\right)
$$

é a matriz da segunda translação. Substituindo-se (3) em (5), temos

$$
\left(\begin{array}{c}
z_{1} \\
z_{2} \\
z_{3} \\
1
\end{array}\right)=\left(\begin{array}{cccc}
1 & 0 & 0 & -b_{1} \\
0 & 1 & 0 & -b_{2} \\
0 & 0 & 1 & -b_{3} \\
0 & 0 & 0 & 1
\end{array}\right)\left(\begin{array}{cccc}
1 & 0 & 0 & -a_{1} \\
0 & 1 & 0 & -a_{2} \\
0 & 0 & 1 & -a_{3} \\
0 & 0 & 0 & 1
\end{array}\right)\left(\begin{array}{c}
x_{1} \\
x_{2} \\
x_{3} \\
1
\end{array}\right)
$$

que resulta em

$$
\left(\begin{array}{c}
z_{1} \\
z_{2} \\
z_{3} \\
1
\end{array}\right)=\left(\begin{array}{cccc}
1 & 0 & 0 & -\left(a_{1}+b_{1}\right) \\
0 & 1 & 0 & -\left(a_{2}+b_{2}\right) \\
0 & 0 & 1 & -\left(a_{3}+b_{3}\right) \\
0 & 0 & 0 & 1
\end{array}\right)\left(\begin{array}{c}
x_{1} \\
x_{2} \\
x_{3} \\
1
\end{array}\right)=\left(\begin{array}{c}
x_{1}-\left(a_{1}+b_{1}\right) \\
x_{2}-\left(a_{2}+b_{2}\right) \\
x_{3}-\left(a_{3}+b_{3}\right) \\
1
\end{array}\right)
$$

Neste caso, vemos que a matriz da transformação é dada por

$$
T(a+b)=\left(\begin{array}{cccc}
1 & 0 & 0 & -\left(a_{1}+b_{1}\right) \\
0 & 1 & 0 & -\left(a_{2}+b_{2}\right) \\
0 & 0 & 1 & -\left(a_{3}+b_{3}\right) \\
0 & 0 & 0 & 1
\end{array}\right)=T(b) T(a)
$$

ou seja, a matriz resultante é o produto das matrizes que compõem a transformação, na ordem de realização das transformações, da direita para a esquerda. Assim, se $T(a)$ e $T(b)$ são translações, $T(b) T(a)=T(a+b)$ também é uma translação. Esta propriedade é denominada propriedade de grupo. 
A segunda propriedade é a existência de uma translação que deixa o sistema de coordenadas inalterado. Esta translação é denominada identidade e é representada pela matriz

$$
T(0)=\left(\begin{array}{cccc}
1 & 0 & 0 & 0 \\
0 & 1 & 0 & 0 \\
0 & 0 & 1 & 0 \\
0 & 0 & 0 & 1
\end{array}\right) \equiv \mathbf{1}_{4} .
$$

A identidade representa a translação com todos os parâmetros nulos.

A terceira propriedade é a existência de uma transformação inversa $T^{-1}(a)=T(-a)$, representada pela matriz

$$
T(-a) \equiv\left(\begin{array}{cccc}
1 & 0 & 0 & a_{1} \\
0 & 1 & 0 & a_{2} \\
0 & 0 & 1 & a_{3} \\
0 & 0 & 0 & 1
\end{array}\right)
$$

Obviamente, temos $T(a) T(-a)=T(-a) T(a)=\mathbf{1}_{4}$. As propriedades de grupo, da existência da identidade e do elemento inverso são as características de uma estrutura matemática denominada grupo.

Uma quarta propriedade é especial no caso das translações. A composição de duas translações em sequência é representada pelo produto matricial, enquanto a ordem do produto entre matrizes importa no resultado, o que é conhecido como não comutatividade do produto matricial. Ou seja, se $A$ e $B$ são matrizes, $A B \neq B A$, no geral. Contudo, matrizes de translação comutam entre si, ou seja, $T(a) T(b)=T(b) T(a)$. Dizemos, assim, que uma translação é um elemento de um grupo abeliano. O conjunto de todas as matrizes de translação sobre o espaço euclidiano tridimensional forma o grupo de translações $T_{3}$. Note que o grupo $T_{3}$ é representado por matrizes $4 \times 4$, portanto, dizemos que o grupo tem dimensão 4 .

Dizemos que o espaço $\mathbb{R}^{3}$ é homogêneo pois a métrica euclidiana, que calcula a distância entre pontos, é invariante por translações. Podemos ver isso diretamente da definição

$$
D(x, y)=\sqrt{\left(y_{1}-x_{1}\right)^{2}+\left(y_{2}-x_{2}\right)^{2}+\left(y_{3}-x_{3}\right)^{2}} .
$$

Uma translação dos pontos $x$ e $y$ pelos mesmos parâmetros $\left(a_{1}, a_{2}, a_{3}\right)$, em dois novos pontos $x^{\prime}$, resulta em

$$
\begin{aligned}
D^{\prime}\left(x^{\prime}, y^{\prime}\right) & =\sqrt{\left(y_{1}^{\prime}-x_{1}^{\prime}\right)^{2}+\left(y_{2}^{\prime}-x_{2}^{\prime}\right)^{2}+\left(y_{3}^{\prime}-x_{3}^{\prime}\right)^{2}} \\
& =\sqrt{\left(y_{1}-a_{1}-x_{1}+a_{1}\right)^{2}+\left(y_{2}-a_{2}-x_{2}+a_{2}\right)^{2}+\left(y_{3}-a_{3}-x_{3}+a_{3}\right)^{2}} \\
& =\sqrt{\left(y_{1}-x_{1}\right)^{2}+\left(y_{2}-x_{2}\right)^{2}+\left(y_{3}-x_{3}\right)^{2}} \\
& =D(x, y) .
\end{aligned}
$$

Portanto, as translações deixam invariante a geometria dos espaço euclidiano. Dizemos, assim, que o grupo de translações é uma simetria de $\mathbb{R}^{3}$ e, também, que o espaço $\mathbb{R}^{3}$ é homogêneo.

A homogeneidade do espaço euclidiano, que também pode ser demonstrada em qualquer dimensão, implica na invariância das características geométricas do sistema físico, como suas dimensões, por translações. Assim, dois observadores transladados um com relação ao outro concordarão com as dimensões de um sistema físico. 